VOL. II (1974), 5-9.

\title{
How a rare species might become a pest
}

\section{Phil Diamond}

\begin{abstract}
A model is proposed for. the population growth of a rare species after predation pressure is lifted. A geometric probability argument is used to suggest that, as population density increases, breeding encounters become more frequent and the consequent rate of increase is much steeper than the exponential. This may explain the population explosion of crown-of-thorns starfish recorded recently.
\end{abstract}

The importance of key predators for the maintenance of diversity and stability of marine communities has been conclusively shown [8]. Little attention has been given to the effects of reducing such predator pressure on a species which is nomally rare. Scarcity (and the concomitant lower probability of breeding encounters between adults [1], p. 337) results in a lower per capita rate of population increase than would occur at higher population densities. A model is constructed below for the increase of a rare species. It is shown that the consequent growth of numbers is far greater than the exponential growth expected in the early part of a logistic curve.

The equation that is used to describe prey dynamics is:

$$
d V / d t=a V(1-f(V)-C g(V, C)),
$$

where $V$ is the density of the prey, $C$ that of the predator, and $a$ is a reproductive rate. The subtracted terms pertain to density-dependent negative feedback and the toll taken of the prey through predation (see for

Received 20 February 1974. 
example Pielou [9]). If the predator is removed from the system,

$$
d V / d t=a v(1-f(V)) .
$$

Suppose that each mature adult moves in a circular region of area $r$ and that these are scattered at random over a large expanse $A$, and $r \ll A$. When two such circles overlap, the adults are assumed to encounter one another. If these encounters are infrequent, as would be probable with low population density, the expected number of spawnings will be directly proportional to the expected number of encounters per unit area, $E$. So the rate of increase will depend linearly on the number of encounters, $a=b E$.

It is known that

$$
E=V(1-\exp (-4 r V)),
$$

to a very good approximation if $r \ll A$ [2]. Note that at rare density, the rate of increase $a$ is proportional not merely to $V$, but to $V^{2}$. This produces a very powerful effect.

Major simplifications included in this model are:

(a) the area $r$ of the circles is a deterministic constant rather than a random variable, and the area is circular;

(b) individuals of the species are not attracted to each other over large distances by some pheromone.

If (a) is taken into account, the results are very much the same and the expression (2) has much the same form at low densities $V$, and is still proportional to the square of $V$ [2], [6]. The factor (b) can only accelerate the population explosion as $V$ increases.

As the density and the number of encounters increases, the rate $a$ will no longer depend linearly on $E$. It will approach a limiting value $L$ which is a measure of the greatest possible breeding capacity; at which the infrequency of contact between individuals is no longer a factor. One such rate has been chosen for illustration [3]:

$$
a=L E /(K+E) \text {. }
$$

The equations (1)-(3) can be combined and the solution of the resulting differential equation is expressible in the form $g(V)=t$. 


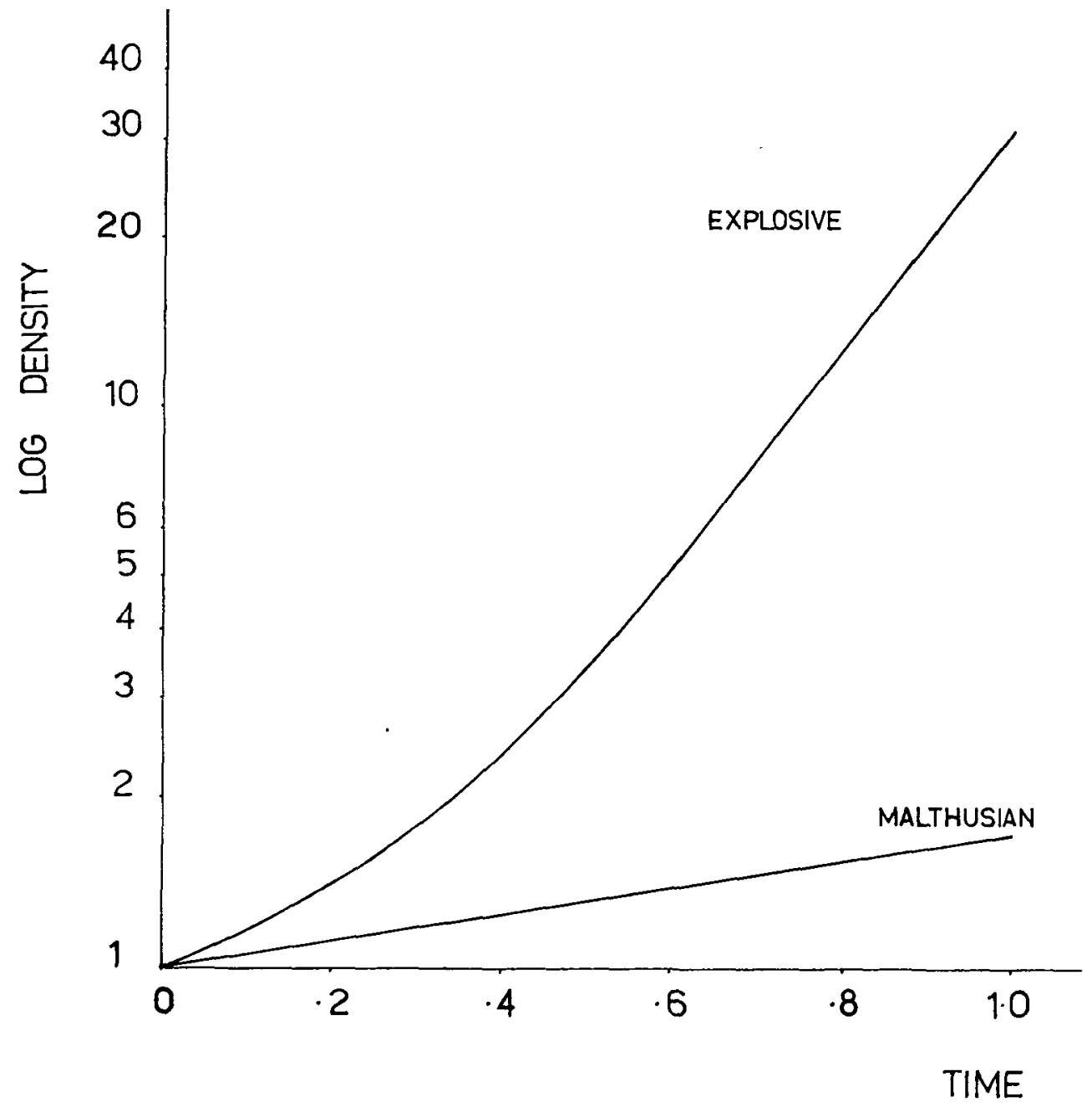

Figure 1. The curve labelled "explosive" is a numerical solution of equation (1) with $L=5, K=0.25, r=0.1$ and an initial density of one individual per unit area. The function $f(V)=V / 200$. The lower, exponential rate is calculated at the initial rate of the explosive increase; $V=\exp (1.73 t)$.

Perhaps more insight into the nature of the solution is gained by numerical solution for given values of the constants. This was done using a fourth order Runge-Kutta method [7], and a typical example is shown in the figure. 
The growth curve is much steeper, in its initial stages, than Malthusian growth (where $a$ is constant). The constants chosen are not crucial and do not affect the qualitative phenomenon of an explosive growth which is much steeper than the exponential growth to be expected from equation (1).

The crown-of-thorns starfish, Acanthaster planci is normally distributed on coral reefs at a low density of six per square kilometre [4]. There is some evidence that the giant triton, Charonia tritonis, is a key predator which has been removed by intensive shell collection [5]. It is suggested, that the mating mechanism described above is a possible explanation of the suddenness of the starfish population explosion on the Barrier Reef and elsewhere.

\section{References}

[1] H.G. Andrewartha and L.C. Birch, The distribution and abundance of animals (University of Chicago Press, Chicago, London, 1954).

[2] P. Armitage, "An overlap problem arising in particle counting", Biometrika 36 (1949), 257-266.

[3] Raymond P. Canale, "An analysis of models describing predator-prey interaction", Biotechnol. and Bioeng. 12 (1970), 353-378.

[4] Robert Endean, "Population explosion of acanthaster planci and associated destruction of hermatypic corals in the Indo-West Pacific region", Biology and geology of coral reefs. Vol. II: Biology 1 (Academic Press, New York, London, 1974).

[5] R. Endean, 2nd Internat. Sympos. Coral Reefs (In press).

[6] C. Mack, "The expected number of clumps when convex laminae are placed at random and with random orientation on a plane area", Proc. Combridge Philos. Soc. 50 (1954), 581-585.

[7] J.M. McCormick and M.G. Salvadori, Numemical methods in FORTRAN (Prentice-Hall, New Jersey, 1965).

[8] R.T. Paine, "A short-term experimental investigation of resource partitioning in a New Zealand rocky intertidal habitat", Ecology 52 (1971), 1096-1106. 
[9] E.C. Pielou, An introduction to mathematical ecology (WileyInterscience [John Wiley \& Sons], New York, London, Sydney, 1969).

Department of Mathematics,

University of Queensland,

St Lucia,

Queensland. 